\title{
Dual and triple tasks performance in institutionalized prefrail and frail older adults
}

\author{
Laura Lorenzo-López ${ }^{1}$, María Campos-Magdaleno², Rocío López-López ${ }^{1}$, David \\ Facal $^{2}$, Arturo X. Pereiro ${ }^{2}$, Ana Maseda ${ }^{1}$, Julia Blanco-Fandiño ${ }^{1}$, José Carlos \\ Millán-Calenti $^{1}$
}

${ }^{1}$ Gerontology and Geriatrics Research Group, Instituto de Investigación biomédica de A Coruña (INIBIC),
Complexo Hospitalario Universitario de A Coruña (CHUAC), SERGAS, Universidade da Coruña, A
Coruña, Spain
2 Department of Developmental Psychology, University of Santiago de Compostela, Santiago de Compostela,
Spain

\begin{abstract}
Objective. The aim of this pilot study was to investigate differences on dual- and triple-task performance in institutionalized prefrail and frail older adults. Performance on these tasks is relevant since many activities of daily living involve simultaneous motor and cognitive tasks.
\end{abstract}

Methods. We used a phenotypic description of frailty based on the presence or absence of five criteria related to physical fitness and metabolism (unintentional weight loss, self-reported exhaustion, muscle weakness, low gait speed, and low physical activity). Thirty-three institutionalized older adults ( $\geq 65$ years, $78.8 \%$ females) were divided according to their frailty status. Participants completed cognitive tasks (a phonemic verbal fluency task and a visuospatial tracking task) while cycling on a stationary cycle (upper- and lower-extremity function was assessed). Cycling (number of arm and foot cycles) and cognitive (number of correct answers) performances were measured during single-, dual-, and triple-task conditions. Performances and costs of dual -and triple- tasking on cycling and cognitive performances were compared between prefrail and frail groups.

Result. Prefrail and frail older adults did not differ in their performance in dual-tasks; however, frail older adults showed a poorer performance in the triple-task.

Conclusions. Although future studies need to confirm our observations in larger samples, this pilot study suggests that developing new tools based on triple tasking could be useful for the comprehensive assessment of frailty.

Keywords

Cognitive aging, dual-tasks, frail older adults, multiple-tasks, triple-tasks. 


\section{Introduction}

Performing multiple tasks at the same time has been a largely studied topic in exploring the limits of human performance.1, 2 Dual-tasking (DT) is the most used paradigm, consisting in executing two tasks simultaneously, generally combining a sensorimotor with a cognitive one. This design allows the comparison of single- and multiple-performance of the same tasks, obtaining information of the motor and/or cognitive interference.3-5 Performance costs can occur in one of the tasks, or both of them in different proportions. Additionally, subjects can prioritize intentionally one specific task to achieve better execution, especially when the experimental conditions are more demanding. As a general trend, performance declines and costs increase gradually as task demands rises6, 7 in all ages, but especially in older adults. DT affects mobility and cognitive performance in older adults compared to young adults.8 There is no consensus in age-related differences in DT costs,1, 9, 10 but the literature provides broad evidence of higher difficulties in DT compared to single tasks in cognitive unimpaired1, 11, 12 and impaired older adults.7, 11, 13 Cognitive status has proven to be the source of main differences in DT costs, with larger interferences in initial stages of cognitive decline5, 7, 9, 14 and dementia15-17 than in cognitive unimpaired participants. Thus, some authors suggested that DT may be sensitive to transitions in cognitive status through aging7, 18, 19 and could be able to predict progression to dementia.20, 21

Frailty is a highly prevalent geriatric syndrome associated with a high risk of hospitalization, falls, institutionalization, and mortality in older adults.22 Although the frailty concept is widely accepted, its operational definition remains controversial, being the physical phenotypic 22 and the deficit accumulation23 complementary models commonly used in geriatric medicine. The phenotypic model operationalizes frailty as a biological syndrome,22 whereas the deficit accumulation model defines frailty as a multidimensional risk state.23 Beyond the original concept of frailty, which is mostly defined by physical status, more recent definitions include the role of cognitive function in the frailty status, 24 since frailty together with cognitive impairment or cognitive frailty are shown to be strong and independent predictors of cognitive decline over time. Recent research have studied the role of multiple-tasks in the relationship between frailty, gait, and cognition.16, 25

Motor dual-task Timed Up \& Go (TUG) tests seem to be more valid and sensitive than single TUG in identifying frail and prefrail community-dwelling middle-aged and older individuals.26, 27 DT-related gait changes were correlated with polymedication and impaired mobility in transitional frail older adults.28 It is important to note that in these studies,16, 25-27 frailty status was operationalized based on the Fried's phenotypic definition,22 which may be problematic since gait speed is one of the five criteria included in the phenotype itself.

As a variant of the multiple-task paradigm, the triple-task (TT) design allows studying performance differences into a more demanding condition by including different combinations of three sensorimotor and cognitive tasks. In community-dwelling older adults, higher costs in walking tasks were found in TT compared with that of DT29, 30 Not only multiple-task cost but also prioritization tendencies can be differentially found in TT compared with DT. In this regard, when older participants are forced to perform above their "resource threshold," natural prioritization tends to be the sensorimotor over the cognitive tasks.31 Doumas and Krampe6 observed higher costs in a working memory cognitive task in a TT than in a DT paradigm, with older adults prioritizing postural control over memory performance. As far as we know, TT paradigms have not been applied in institutionalized frail older adults.

Recent literature has focused on the study of the relationship between physical frailty and cognitive decline and its underlying mechanisms; however, little is known about how much these aspects influence the motor-cognitive performance on multi-task performance. The purpose of the current pilot study was to explore differences in DT and TT performance in institutionalized 
prefrail and frail older adults. Impairments in both mobility and cognition are common in frail institutionalized older adults, making them an important population for the clinical use of multitask testing. We hypothesized that frail participants would present worse performance on DT and TT contexts due to the changes in physical and cognitive functions. Performance on these tasks is clinically relevant because most activities of daily living involve the simultaneous performance of two or more motor and cognitive tasks. Acquiring more information about DT and TT performance in older adults can be clinically significant since these paradigms allow screening for both cognitive and motor performance and identify both cognitive and physical frailty in older adults.

\section{Methods}

\subsection{Participants}

Participants were recruited from the Gerontological Complex La Milagrosa sited in A Coruña (Spain), a long-term care center with capacity for 70 users in a daycare setting and 64 institutionalized users in a nursing home. Individuals with mobility disorders in upper or lowerextremities, aphasia, or other neurological disorders that could affect mobility or cognition, and those with Global Deterioration Scale (GDS)32 $\geq 5$ were excluded. Thirty-three institutionalized older adults from the nursing home (age 65 years or older; mean age $=83.21 \pm 7.39$, range 68-79; $78.8 \%$ females) fulfilled the inclusion criteria of having no severe cognitive decline. They were divided as a function of their frailty status (prefrail group with 1 or 2 frailty criteria, $n=15,45.5 \%$; frail group with $\geq 3$ criteria, $\mathrm{n}=18,54.5 \%$, none subject was robust).

The study was approved by the Ethics Committee of the University of A Coruña and was in conformity with the principles embodied in the World Medical Association's Declaration of Helsinki. All subjects were informed in advance about the study and gave their consent to participate in the study, either directly or through their legal representatives.

\subsection{Tasks and procedure}

Participants were required to perform a static cycling task including both upper-extremity and lower-extremity motion/function. Regarding cognitive tasks, we selected a phonemic verbal fluency task and a paper-and-pencil tracking (circle crossing) task, which can be performed simultaneously in the DT and TT experimental conditions and they are easy to use in both research and clinical settings. Verbal fluency tests require a listing of words that begin with a prespecified letter. The letters $\mathrm{P}, \mathrm{R}$, and $\mathrm{M}$ were used. To avoid practice effects, these letters were counterbalanced in single, double, or triple conditions between participants. The tracking task requires to draw a line through circles arranged in a path around a sheet of paper.9

All participants underwent a first assessment session, which involved the collection of demographic (age, gender, education) and health status (comorbidity,33 number of daily medications) data, and screening of frailty status. Participants' cognitive status was also assessed by the GDS32 and the Spanish version34 of the Mini-Mental State Examination (MMSE).35 No more than 15 days later, a second session was carried out performing DT and TT. A qualified clinical psychologist of the long-term care center carried out the first session, and trained nurses and psychologists carried out the second session.

During the first session, frailty status of each participant was assessed according to the presence or absence of five specific phenotypic criteria proposed by Fried et al22: (a) Shrinkingunintentional weight loss of at least $4.5 \mathrm{~kg}$ in prior year; (b) Self-reported exhaustion-identified by two questions from the Spanish version36 of the Center for Epidemiological Studies- 
Depression scale37; (c) Muscle weakness-grip strength in the lowest $20 \%$ at baseline, adjusted for gender and body mass index; (d) Slow walking speed-the slowest $20 \%$ at baseline, based on time to walk 15 feet, adjusting for gender and height; and (e) Low physical activity - the lowest $20 \%$ at baseline, based on a weighted score of kilocalories expended per week, calculated according to the Spanish validation38 of the Minnesota Leisure Time Activity questionnaire,39 and adjusted by gender. Participants were classified as frail if they met three or more of the criteria, prefrail if they met one or two of the criteria, and robust if they met none of them. These phenotype criteria constitute a common and validated reference frame for geriatric studies. Finally, a 5-point Likert scale was also used to gather information about the familiarization with cycling (never, rarely, occasionally, frequently, and very frequently).

During the second session, all the tasks were conducted first in single conditions: (a) Fluency single-task, (b) Tracking single-task, (c) Arm cycling single-task, (d) Foot cycling single-task, and then in dual- and triple conditions: (a) Fluency-tracking DT, (b) Fluency-arm cycling DT, (c) Fluency-foot cycling DT, (4d) Fluency-tracking-foot cycling TT. Both arm and foot cycling was performed on a stationary and portable pedal exerciser (M-bike II, Body Care) against minimal resistance (single-task condition). Before conducting the cycling tasks, we ensured the pedal exerciser was calibrated and working properly, and verified that the participants was dressed appropriately for arm and foot cycling. Then a supervised practice trial was carried out, providing a detailed explanation about how to use the exerciser (ensuring the proper technique and a full range of motion), and allowing time to familiarize with the required position and cycling tasks. Immediately before the cycling tasks, the number of cycles completed during the single-task condition was collected while participants cycled for 1 minute at a self-selected comfortable rate.

In the dual conditions, the participants performed the same cycling task while engaging in the cognitive tasks simultaneously for one-minute, and performed the cognitive tasks simultaneously. The order of the tasks for the single and dual conditions was counterbalanced. Finally, the participants who were able to successfully perform the foot cycling, the verbal fluency and the tracking tasks in single and dual conditions were invited to perform these tasks simultaneously during 1 minute, in a TT paradigm. Participants were asked to perform all the tasks as well as possible, with no instructions prioritizing one task over the others both in DT and TT paradigms.

\subsection{DT and TT cost measurements}

The proportional DT costs (eg, the reduction of the performance under DT conditions relative to the single-task condition) on cognition and cycling were quantified with the following formula40: DT cost $=[(\mathrm{DT}$ score $-\mathrm{ST}$ score $) / \mathrm{ST}$ score $] \times 100 \%$. The proportional TT costs $(\mathrm{eg}$, the reduction of the performance under TT conditions relative to the single-task condition) were quantified with the formula: TT cost $=[($ TT score - ST score $) /$ ST score $] \times 100 \%$. For the physical costs computation, the number of cycles was used, whereas for the cognitive costs, the number of words and the number of tracked circles was used to compare single and DT or TT performances. Negative values indicate a worse performance during the dual- and triple-conditions.

\subsection{Statistical analysis}

The SPSS statistical software package (version 20.0) was used to analyze all of the data. Descriptive results are reported as the mean and SD. In order to explore whether DT and TT performances and costs differed based on frailty level (defined by Fried's phenotype), the nonparametric Mann-Whitney $U$ test was used to compare frail and prefrail groups. The level of statistical significance was set at $\mathrm{P}<.05$. 


\section{Results}

Demographic and health-related characteristics of the prefrail and frail groups are shown in Table 1. No significant differences between the frailty groups were observed for demographic and health information, including age, gender, education, time of institutionalization, MMSE score, number of medications, comorbidity, and previous cycling experience.

Table 1. Participants characteristics as a function of frailty status

\begin{tabular}{|c|c|c|c|}
\hline & $\begin{array}{l}\text { Frailty status } \\
\text { Prefrailty }(\mathrm{n}=15)\end{array}$ & Frailty $(n=18)$ & P-value \\
\hline Age (years), mean (SD) & $82.87(8.40)$ & $83.50(6.67)$ & .899 \\
\hline Gender, n (\%) & & & .876 \\
\hline Female & $12(80.0 \%)$ & $14(77.8 \%)$ & \\
\hline Male & $3(20.0 \%)$ & $4(22.2 \%)$ & \\
\hline Education (years), mean (SD) & & & .741 \\
\hline$\leq 8$ & $10(66.7 \%)$ & $14(77.8 \%)$ & \\
\hline $9-17$ & $3(20.0 \%)$ & $2(11.1 \%)$ & \\
\hline$>17$ & $2(13.3 \%)$ & $2(11.1 \%)$ & \\
\hline Time of institutionalization (months), mean (SD) & $21.13(34.16)$ & $14.17(21.89)$ & .254 \\
\hline MMSE score, mean (SD) & 21.53 (3.69) & $20.67(4.38)$ & .637 \\
\hline Number of drugs, mean (SD) & $10.13(4.72)$ & $9.11(2.91)$ & .662 \\
\hline Comorbidity, n (\%) & & & .270 \\
\hline No comorbidity & $11(73.3 \%)$ & $11(61.1 \%)$ & \\
\hline Low comorbidity & $1(6.7 \%)$ & $5(27.8 \%)$ & \\
\hline High comorbidity & $3(20.0 \%)$ & $2(11.1 \%)$ & \\
\hline Cycling frequency, n (\%) & & & .839 \\
\hline Never & $4(26.7 \%)$ & $5(27.8 \%)$ & \\
\hline Rarely & $2(13.3 \%)$ & $1(5.6 \%)$ & \\
\hline Occasionally & $2(13.3 \%)$ & $4(22.2 \%)$ & \\
\hline Frequently & $1(6.7 \%)$ & $1(5.6 \%)$ & \\
\hline Very frequently & $1(6.7 \%)$ & $4(22.2 \%)$ & \\
\hline N/A & $5(33.3 \%)$ & $3(16.6 \%)$ & \\
\hline
\end{tabular}

Abbreviations: MMSE, Mini-Mental State Examination. 


\subsection{Performance under single, DT, and TT conditions}

Group differences (prefrail vs frail) in single and DTs performance and proportional DT costs are shown in Table 2 and Figure 1.

Table 2. Mean and SDs (between brackets) and group differences in single-, dual-, and triple-tasks performance

\begin{tabular}{|c|c|c|c|c|}
\hline & & $\begin{array}{l}\text { Frailty status } \\
\text { Prefrailty }\end{array}$ & Frailty & Z-value \\
\hline \multirow[t]{9}{*}{ Fluency-tracking $(\mathrm{n}=26)$} & & $(n=13)$ & $(\mathrm{n}=13)$ & \\
\hline & Age (years) & $82.92(8.67)$ & $84.00(5.92)$ & -0.05 \\
\hline & MMSE Score & $21.77(3.90)$ & $21.38(4.43)$ & -0.13 \\
\hline & Fluency (single) & $4.69(3.26)$ & 5.00 (3.29) & -0.21 \\
\hline & Fluency (dual) & $3.77(3.06)$ & $4.46(4.31)$ & -0.08 \\
\hline & Dual-task Cost & $-1.40(79.87)$ & $-8.53(92.47)$ & -0.54 \\
\hline & $\begin{array}{l}\text { Tracking } \\
\text { (single) }\end{array}$ & $36.69(22.75)$ & $34.00(18.04)$ & -0.03 \\
\hline & Tracking (dual) & $23.62(22.56)$ & $18.77(16.83)$ & -0.23 \\
\hline & Dual-task Cost & $-40.86(24.65)$ & $-45.06(34.70)$ & -0.69 \\
\hline \multirow[t]{10}{*}{ Fluency-arm cycling $(\mathrm{n}=27)$} & & $(n=14)$ & $(n=13)$ & \\
\hline & Age (years) & $83.36(8.49)$ & $84.23(5.81)$ & -0.15 \\
\hline & MMSE Score & 21.29 (3.69) & $20.92(4.55)$ & -0.19 \\
\hline & Fluency (single) & $5.86(4.83)$ & $4.69(3.47)$ & -0.59 \\
\hline & Fluency (dual) & 5.36 (3.69) & $4.23(3.90)$ & -0.86 \\
\hline & Dual-task Cost & $18.79(116.72)$ & $-3.08(101.52)$ & -0.71 \\
\hline & $\begin{array}{l}\text { Cycling-upper } \\
\text { (single) }\end{array}$ & $64.00(27.63)$ & $59.15(30.75)$ & -0.51 \\
\hline & $\begin{array}{l}\text { Cycling-upper } \\
\text { (dual) }\end{array}$ & $46.43(25.37)$ & $37.46(25.08)$ & -0.90 \\
\hline & Dual-task Cost & $-29.19(20.03)$ & $-38.51(28.95)$ & -1.36 \\
\hline & & $(\mathrm{n}=10)$ & $(n=9)$ & \\
\hline \multicolumn{5}{|l|}{ Fluency-foot cycling $(n=19)$} \\
\hline & Age (years) & $85.50(8.10)$ & $81.56(4.53)$ & -1.72 \\
\hline & MMSE Score & $22.6(3.81)$ & $20.11(4.57)$ & -1.23 \\
\hline & Fluency (single) & 5.20 (3.39) & $3.44(1.51)$ & -1.16 \\
\hline & Fluency (dual) & $7.70(5.66)$ & 2.89 (2.89) & -2.14 \\
\hline
\end{tabular}




\begin{tabular}{|c|c|c|c|c|}
\hline & Dual-task Cost & 86.05 (158.79) & $-15.56(94.62)$ & -1.84 \\
\hline & $\begin{array}{l}\text { Cycling-down } \\
\text { (single) }\end{array}$ & $57.40(31.33)$ & $45.33(29.15)$ & -0.82 \\
\hline & $\begin{array}{l}\text { Cycling-down } \\
\text { (dual) }\end{array}$ & 49.90 (28.59) & $51.22(39.87)$ & -0.20 \\
\hline & Dual-task Cost & $-8.41(33.94)$ & 38.36 (157.16) & -0.25 \\
\hline \multirow{12}{*}{$\begin{array}{l}\text { Fluency-tracking-foot cycling } \\
(\mathrm{n}=13)\end{array}$} & & $(\mathrm{n}=6)$ & $(\mathrm{n}=7)$ & \\
\hline & Age (years) & $85.50(8.10)$ & 81.29 (5.19) & -0.13 \\
\hline & MMSE Score & $23.33(4.50)$ & $21.43(4.31)$ & -0.72 \\
\hline & Fluency (single) & $7.33(2.58)$ & $4.00(1.15)$ & $-2.65^{* *}$ \\
\hline & Fluency (triple) & $4.83(3.06)$ & 2.43 (1.99) & -1.44 \\
\hline & $\begin{array}{l}\text { Triple-task } \\
\text { Costs }\end{array}$ & $-35.03(43.90)$ & $-27.62(66.96)$ & -0.07 \\
\hline & $\begin{array}{l}\text { Tracking } \\
\text { (single) }\end{array}$ & 46.83 (15.68) & 34.43 (19.97) & -1.14 \\
\hline & $\begin{array}{l}\text { Tracking } \\
\text { (triple) }\end{array}$ & $42.00(22.56)$ & $17.86(18.22)$ & $-1.95^{*}$ \\
\hline & $\begin{array}{l}\text { Triple-task } \\
\text { Costs }\end{array}$ & $-5.07(44.98)$ & $-55.27(29.76)$ & $-2.14^{*}$ \\
\hline & $\begin{array}{l}\text { Foot Cycling } \\
\text { (single) }\end{array}$ & $58.00(27.51)$ & $42.71(23.45)$ & -1.00 \\
\hline & $\begin{array}{l}\text { Foot Cycling } \\
\text { (triple) }\end{array}$ & 26.33 (18.77) & 16.57 (16.84) & -0.72 \\
\hline & $\begin{array}{l}\text { Triple-task } \\
\text { Costs }\end{array}$ & $-49.81(33.71)$ & $-7.54(169.84)$ & -0.43 \\
\hline
\end{tabular}

Abbreviations: MMSE, Mini-Mental State Examination.

Notes: Prefrail older adults showed a triple-task cost of $5.07 \%$ on tracking while frail older adults showed a $55.27 \%$ cost. $* \mathrm{P}<.05$. 
(A)

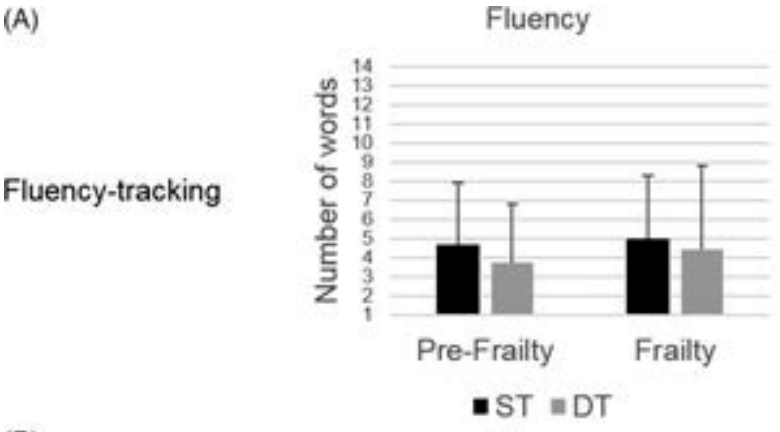

(B)

(C)

Fluency-foot cycling
Fluency

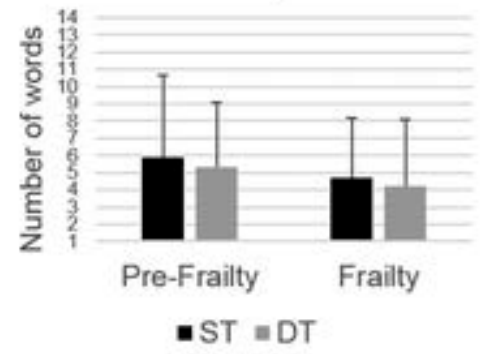

Fluency

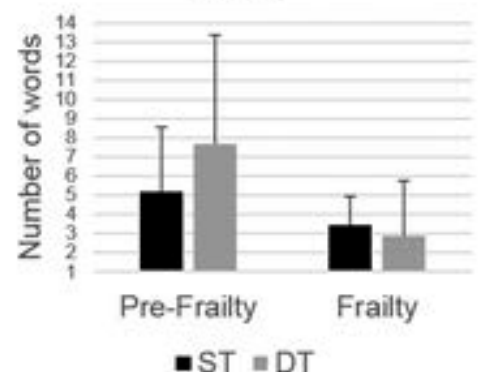

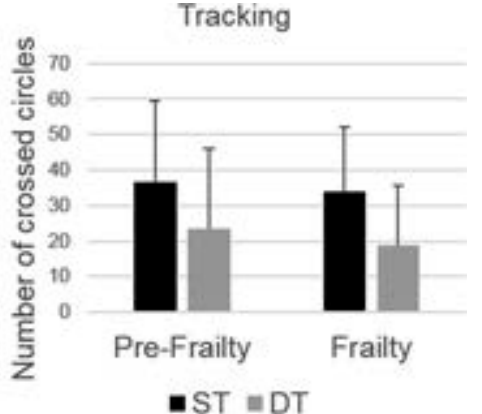

mST $=\mathrm{DT}$ Arm Cycling

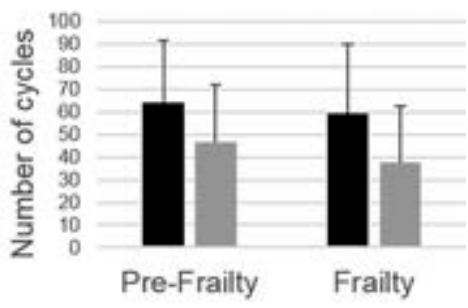

- $\mathrm{ST}=\mathrm{DT}$

Foot Cycling

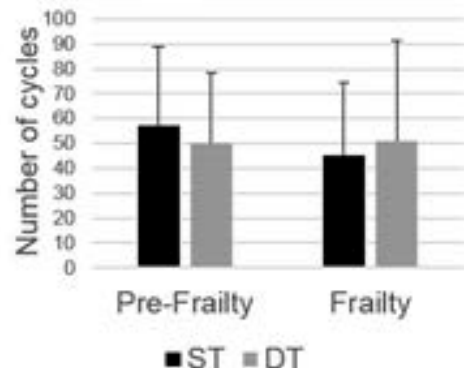

Figure 1. Mean and SD of performances in single and dual-tasks in (A) fluency-tracking, (B) fluency-arm cycling, and (C) fluency-foot cycling conditions, for each group (prefrail and frail)

As shown, results in the DT condition show a general tendency to better performance in prefrail than in frail participants. Nevertheless, no differences were found comparing frailty status. In fact, in the fluency-tracking condition ( $\mathrm{n}=26,13$ prefrail and 13 frail), DT reduced the number of words and the number of crossed circles compared to single conditions in both prefrail $(4.69 \pm 3.26$ vs $3.77 \pm 3.06$ words, $36.69 \pm 22.75$ vs $23.62 \pm 22.56$ circles $)$ and frail participants $(5.00 \pm 3.29$ vs $4.46 \pm 4.31$ words, $34.00 \pm 18.04$ vs $18.77 \pm 16.83$ circles $)$, with no significant differences in the between-groups DT costs $(Z=-0.54$ and $Z=-0.69$, respectively). In the fluency-arm cycling condition ( $n=27,14$ prefrail and 13 frail), DT reduced the number of words and the number of arm cycles compared to single conditions in both prefrail (5.86 \pm 4.83 vs $5.36 \pm 3.69$ words, $64.00 \pm 27.63$ vs $46.43 \pm 25.37$ arm cycles) and frail ( $4.69 \pm 3.47$ vs $4.23 \pm 3.90$ words, $59.15 \pm 30.75$ vs $37.46 \pm 25.08$ arm cycles) participants, with no significant differences in the between-groups DT costs $(Z=-0.71, Z=-1.36$, respectively). This decline in performance during DT is greater in arm cycling than in fluency, suggesting that both prefrail and frail older adults prioritize cognition over mobility during DT.

Group differences in single and TTs performance are shown in Table 2 and Figure 2. 

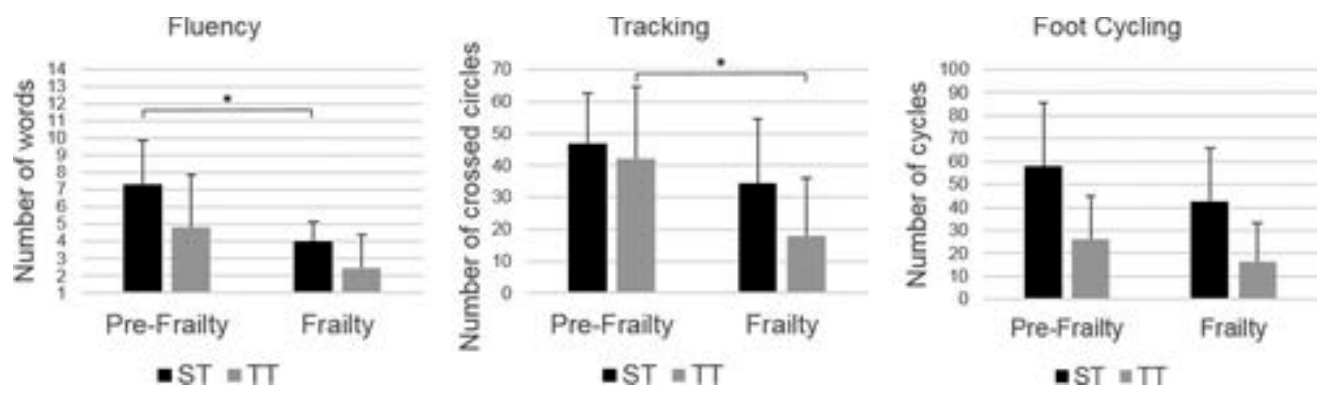

Figure 2. Mean and SD of performances in single and triple-tasks, for each group (prefrail and frail)

Results in the TT show the same tendency, with better performances in prefrail than in frail participants. Regarding the frailty status, significant differences were found for the fluency score in the single condition. This difference was not present in the trial condition. Participants who completed the TT and those who did not complete the task did not significantly differ in age ( $\mathrm{Z}=$ $-0.48, \mathrm{P}=.631)$, gender $(\mathrm{P}=.509)$ or education level $(\mathrm{P}=.749)$.

During triple tasks, participants reduced the number of words, the number of crossed circles, and the number of foot cycles compared to single conditions in both prefrail $(7.33 \pm 2.58 \mathrm{vs}$ $4.83 \pm 3.06$ words, $46.83 \pm 15.68$ vs $42.00 \pm 22.56$ tracked circles, $58.00 \pm 27.51$ vs $26.33 \pm 18.77 \mathrm{ft}$ cycles $)$ and frail $(4.00 \pm 1.15$ vs $2.43 \pm 1.99$ words, $34.43 \pm 19.97$ vs $17.86 \pm 18.22$ tracked circles, $42.71 \pm 23.45$ vs $16.57 \pm 16.84$ foot cycles) groups. TT cost in the frail group was statistically significantly higher than in the prefrail group $(\mathrm{U}=6.0, \mathrm{P}=.035)$. Prefrail older adults showed a TT cost of $5.07 \%$ in the tracking task, while frail older adults showed a $55.27 \%$ cost and exhibited considerably higher difficulties to perform the TT.

\section{Discussion}

The main aim of the present study was to investigate potential differences on DT and TT performance as a function of frailty status in institutionalized older adults. To our knowledge, this is the first study exploring performance during TTs in frail and in institutionalized participants. The successful performance of simultaneous tasks is essential for the independence of older adults in general, but it may be especially difficult in institutionalized samples, due to the higher decline of physical and cognitive functions. DTs and TTs may play an important role in daily life, and difficulty to coordinate and distribute attention among concurrent motor and cognitive activities can affect the quality of life especially in frail institutionalized older adults. It has been shown that DT protocols that include cognitive distractions during standing more greatly compromise balance control in frail and prefrail community-dwelling individuals compared to healthy older adults, leading to an increased risk of falls. 41

Most of the previous studies16, 25-27 have explored the effects of different cognitive tasks on gait stability and/or gait speed in frail older adults. We have used an alternative motor task (static cycling) including both upper-extremity and lower-extremity motion/function. Taking into account that we used the Fried phenotype to operationalize frailty status, gait speed would be problematic, being both a study variable and a primary outcome measure. Regarding cognitive tasks, we selected a phonemic verbal fluency task and a paper-and-pencil tracking (circle crossing) task, which can be performed simultaneously in the cognitive-cognitive DT and TT experimental conditions. 
In general, all participants showed poor performances in response to DT by decreasing the number of words, tracked circles, and cycles. The study revealed no significant differences in DT performances between prefrail and frail participants, suggesting that cognitive-motor interference did not differ as a function of frailty status. The DT condition does not differentiate prefrail and frail participants.16 A similar result was obtained by Rossi et al42 that found that, although frail older adults presented a worse performance in the TUG that nonfrail older adults, the DT condition (TUG associated with a motor-cognitive task) did not differentiate frail and nonfrail participants, regardless of cognitive performance. It cannot be discarded that other types of simultaneously cognitive performed tasks, more directly related to executive function, may interfere with the motor task. An arithmetic task, but not a verbal fluency task, has been shown that significantly interfered with lateral gait stability in transitionally frail older adults.43 Because different types of cognitive tasks may result in different patterns of cognitive-motor interference, other motor and cognitive tasks should be included in future studies.

Importantly, frail older adults exhibited difficulties performing the TT (involving the simultaneous performance of fluency, circles tracking, and foot cycling tasks, with higher resource demands) compared with prefrail older adults, which may have important clinical applications. Nevertheless, we should take into account that in the present study cognitive status was measured using the MMSE, with no significant differences between the groups. We cannot, however, discard that the differences seen on the TT paradigm is due to differences in specific cognitive domains. The allocation of attention in concurrent tasks represents executive processes that may be sensitive to the frailty status.28 Cross-sectional and longitudinal studies have shown links between physical frailty and cognitive functions, mainly executive function, attention and processing speed.44 A comprehensive neuropsychological assessment should be included in future studies. Complementarily, differences in fluency performance in the simple condition vs differences in the triple condition in the tracking task can be interpreted in light of prioritization tendencies.6, 31 Prefrail participants show a higher performance in verbal fluency in the simple condition, when all their resources are allocated to perform this task. Nevertheless, in TT conditions these prefrail participants seem to prioritize their cognitive-motor performance in the tracking task over the cognitive-cognitive performance in the fluency task, in line with Doumas and Krampe.6 Natural prioritization of sensorimotor over cognitive tasks31 would be crucial in avoiding falling risks, but it could also lead to a greater tendency to avoid cognitively stimulating contexts.

It has been shown that DT performance can be modified with specific DT interventions. Recently, a 6-week high-speed lower extremity stationary cycling program has shown to be effective to improve rapid movement and neuromuscular function in older adults45 especially related to activities of daily living and important for fall prevention. In the same line, a supervised DT program was effective in the attenuation of frailty (improving gait speed performance) in nursing home residents.46 Our findings improve our understanding of frailty-related differences in DT and TT performances, which may have clinical implications for developing future low-cost preventive intervention programs to minimize difficulties in multitask performance in nursing homes.

\section{Conclusion}

All participants showed poorer performances in response to multiple-tasks by decreasing the number of words, tracked circles and cycles compared with single-tasks. Pre-frail and frail older adults did not differ in DT costs, whereas TT cost in the frail group was statistically significantly higher than in the prefrail group. These results highlight the relevance of multiple tasking in both assessment and intervention.

Our study has several limitations. First, the size of the sample was small, and the SDs were high, with relatively high dispersion in outcomes. Because the optimal sample size was not estimated, we cannot ensure sufficient power to extrapolate the statistical results to the overall 
population, and our findings should be interpreted with caution. Second, because of the exclusion criteria, the frailest individuals have probably not been included in the study and this could affect the generalization of our findings to the institutionalized population. Third, due to the crosssectional nature of the present study, no causal relationships between the frailty state of the participants and the observed findings under TT conditions can be drawn. Finally, it is also unclear how the use of an alternative definition of frailty would have influenced our findings. Thus, future longitudinal studies with higher sample sizes are needed to assess whether a poor performance in TT may predict the onset of frailty syndrome and related adverse outcomes.

\section{Acknowledgements}

The authors acknowledge the professional staff of the Gerontological Complex La Milagrosa and study participants for their time, effort, and dedication. This work was supported by Xunta de Galicia (ED431C 2017/49, ED431F 2017/09, and Frailty Network IN607C 2016/08). Laura Lorenzo-López is supported by the "Ramon y Cajal" Postdoctoral Senior Grant (RYC-201518 394) from the Spanish Ministry of Economy, Industry and Competitiveness, co-financed by the European Social Fund.

\section{References}

1. Bherer L, Kramer AF, Peterson MS, Colcombe S, Erickson K, Becic E. Training effects on dual-task performance: are there age-related differences in plasticity of attentional control? Psychol Aging. 2005; 20(4): 695- 709.

2. Hazeltine E, Teague D, Ivry R. Simultaneous dual-task performance reveals parallel response selection after practice. J Exp Psychol Hum Percept Perform. 2002; 28(3): 527- 545.

3. Falbo S, Condello G, Capranica L, Forte R, Pesce C. Effects of physical-cognitive dual task training on executive function and gait performance in older adults: a randomized controlled trial. Biomed Res Int. 2016; 5812092.

4. Metzger FG, Hobert MA, Ehlis AC, et al. Dual tasking for the differentiation between depression and mild cognitive impairment. Front Aging Neurosci. 2016; 8: 235.

5. Montero-Odasso M, Muir SW, Speechley M. Dual-task complexity affects gait in people with mild cognitive impairment: the interplay between gait variability, dual tasking, and risk of falls. Arch Phys Med Rehabil. 2012; 93(2): 293- 299.

6. Doumas M, Krampe RT. Ecological relevance determines task priority in older adults' multitasking. J Gerontol Ser B Psychol Sci Soc Sci. 2013; 70(3): 377- 385.

7. Holtzer R, Burright RG, Donovick PJ. The sensitivity of dual-task performance to cognitive status in aging. J Int Neuropsychol Soc. 2004; 10(2): 230- 238.

8. Brustio PR, Magistro D, Zecca M, Rabaglietti E, Liubicich ME. Age-related decrements in dual-task performance: comparison of different mobility and cognitive tasks. A cross sectional study. PLoS One. 2017; 12(7):e0181698.

9. Della Sala S, Foley JA, Beschin N, Allerhand M, Logie RH. Assessing dual-task performance using a paper-and-pencil test: normative data. Arch Clin Neuropsychol. 2010; 25(5): 410419.

10. Sebastian MV, Mediavilla R. Does dual-task coordination performance decline in later life? Psichothema. 2017; 29(2): 223- 228.

11. Gillain S, Warzee E, Lekeu F, et al. The value of instrumental gait analysis in elderly healthy, MCI or Alzheimer's disease subjects and a comparison with other clinical test used in single and dual-task conditions. Ann Phys Rehabil Med. 2009; 52(6): 453- 474.

12. Verghese J, Wang C, Lipton RB, Holtzer R, Xionan W. Quantitative gait dysfunction and risk cognitive decline and dementia. J Neurosurg Psych. 2007; 78(9): 929- 935.

13. Perrochon A, Kemoun G, Watelain E, Berthoz A. Walking Stroop carpet: an innovative dualtask concept for detecting cognitive impairment. Clin Interv Aging. 2013; 8: 317- 328. 
14. Makizako H, Doi T, Shimada H, et al. Does a multicomponent exercise program improve dual-task performance in amnestic mild cognitive impairment? A randomized controlled trial. Aging Clin Exp Res. 2012; 24(6): 640- 646.

15. Baddeley AD, Baddeley HA, Bucks RS, Wilcock GK. Attentional control in Alzheimer's disease. Brain. 2001; 124: 1492- 1508.

16. Cadore EL, Casas-Herrero E, Zambom-Ferraresi F, et al. Do frailty and cognitive impairment affect dual-task cost during walking in the oldest old institutionalized patients? Age (Dordr). 2015; 37(6):124.

17. Logie RH, Cocchini G, Della-Sala S, Baddeley AD. Is there a specific executive capacity for dual task coordination? Evidence from Alzheimer's disease. Neuropsychology. 2004; 18(3): 504- 513.

18. Killane I, Fearon C, Newman L, et al. Dual motor-cognitive virtual reality training impacts dual-task performance in freezing of gait. IEEE J Biomed Inform. 2015; 19(6): 1855- 1861.

19. MacAulay R, Wagner MT, Szeles D, Milano NJ. Improving sensitivity to detect mild cognitive impairment: cognitive load dual-task gait speed assessment. J Int Neuropsychol Soc. 2017; 23(6): 493- 501.

20. MacPherson SE, Parra MA, Moreno S, Lopera F, Della-Salla S. Dual task abilities as a possible preclinical marker of Alzheimer's disease in carriers of the e280a presenilin-1 mutation. J Int Neuropsychol Soc. 2012; 18(2): 234- 241.

21. Montero-Odasso MM, Sarquis-Adamson Y, Speechley M, et al. Association of dual-task gait with incident dementia in mild cognitive impairment. Results from the gait and brain study. JAMA Neurol. 2017; 74(7): 857- 865.

22. Fried LP, Tangen CM, Waltson J, et al. Frailty in older adults: evidence for a phenotype. $\mathrm{J}$ Gerontol A Biol Sci Med. 2001; 56(3): M146- M156.

23. Rockwood K, Mitnitski A. Frailty defined by deficit accumulation and geriatric medicine defined by frailty. Clin Geriatr Med. 2011; 27: 17- 26.

24. Facal D, Maseda A, Pereiro AX, et al. Cognitive frailty: a conceptual systematic review and an operational proposal for future research. Maturitas. 2018; 121: 48- 56.

25. Martínez-Ramírez A, Martinikorena I, Lecumberri P, et al. Dual task gait performance in frail individuals with and without mild cognitive impairment. Dement Geriatr Cogn Disord. 2016; 42(1-2): 7- 16.

26. Tang PF, Yang HJ, Peng YC, Chen HY. Motor dual-task timed up \& go test better identifies prefrailty individuals than single-task timed up \& go test. Geriatr Gerontol Int. 2015; 15(2): 204- 210.

27. Guedes RC, Dias RC, Pereira LSM, Silva SLA, Lustosa LP, Dias JMD. Influence of dual task and frailty on gait parameters of older community-dwelling individuals. Braz J Phys Ther. 2014; 18(5): 445- 452.

28. Beauchet O, Dubost V, Herrmann F, Rabilloud M, Gonthier R, Kressig RW. Relationship between dual-task related gait changes and intrinsic risk factors for falls among transitional frail older adults. Aging Clin Exp Res. 2005; 17(4): 270- 275.

29. Laessoe U, Hoeck HC, Simonsen O, Voigt M. Residual attentional capacity amongst young and elderly during dual and triple task walking. Hum Mov Sci. 2008; 27(3): 496- 512.

30. Jeon S, Kim C, Song S, Lee G. Changes in gait pattern during multitask using smartphones. Work. 2015; 53(2): 241- 247.

31. Li KZH, Krampe RT, Bondar A. An ecological approach to studying aging and dual-task performance. In: RW Engle, G Sedek, U vonHecker, DN McIntosh, eds. Cognitive Limitations in Aging and Psychopathology. Cambridge, UK: Cambridge University Press; 2005: 190- 218.

32. Reisberg B, Ferris SH, De Leon MJ, Crook T. The global deterioration scale for assessment of primary degenerative dementia. Am J Psychiatry. 1982; 139(9): 1136- 1139.

33. Charlson ME, Pompei P, Ales KL, Mackenzie CR. A new method of classifying prognostic comorbidity in longitudinal studies: development and validation. J Chronic Dis. 1987; 40: 373- 383.

34. Blesa R, Pujol M, Aguilar M, et al. Clinical validity of the 'mini-mental state' for Spanish speaking communities. Neuropsychologia. 2011; 39(11): 1150- 1157.

35. Folstein MF, Folstein SE, McHugh PR. "Mini-mental state". A practical method for grading the cognitive state of patients for the clinician. J Psychiatr Res. 1975; 12(3): 189- e198.

36. Ruiz-Grosso P, Loret de Mola C, Vega-Diemstmaier J, et al. Validation of the Spanish Center for Epidemiological Studies Depression and Zung Self-Rating Depression Scales: a comparative validation study. PLoS One. 2012; 7(10): e45413. 
37. Radloff LS. The CES-D scale: a self-report depression scale for research in the general population. Appl Psychol Measur. 1977; 1: 385- 401.

38. Ruiz-Comellas A, Pera G, Baena Díez JM, et al. Validación de una versión reducida en Español del cuestionario de actividad física en el tiempo libre de MINNESOTA (VREM). Rev Esp Salud Publica. 2012; 86(5): 495- 508.

39. Taylor HL, Jacobs DR, Schucker B, Knudsen J, Leon AS, Debacker G. A questionnaire for the assessment of leisure time physical activities. J Chronic Dis. 1978; 31(12): 741- 755.

40. McIsaac TL, Lamberg EM, Muratori LM. Building a framework for dual task taxonomy. Biomed Res Int. 2015; 2015: 1- 10.

41. Kang HG, Costa MD, Priplata AA, et al. Frailty and the degradation of complex balance dynamics during a dual-task protocol. J Gerontol Med Sci. 2009; 64(12): 1304- 1311.

42. Rossi PG, Pires de Andrade L, Hotta Ansai J, et al. Dual-task performance: influence of frailty, level of physical activity, and cognition. J Geriatr Phys Ther. 2019; 42(3): E142E147.

43. Beauchet O, Dubost V, Kressig RW. Dual-task-related gait changes in transitionally frail older adults: the type of the walking-associated cognitive task matters. Gerontology. 2005; 51(1): 48- 52.

44. Robertson DA, Savva GM, Kenny RA. Frailty and cognitive impairment. A review of the evidence and causal mechanisms. Ageing Res Rev. 2013; 12: 840- 851.

45. Bellumori M, Uygur M, Knight CA. High-speed cycling intervention improves ratedependent mobility in older adults. Med Sci Sports Exerc. 2017; 49(1): 106- 114.

46. Rezola-Pardo C, Arrieta H, Gil SM, et al. A randomized controlled trial protocol to test the efficacy of a dual-task multicomponent exercise program in the attenuation of frailty in longterm nursing home residents: aging-ON dual-task study. BMC Geriatr. 2019; 19(1):6. 